\title{
Analysis and Performance Evaluation of Entropic Thresholding Image Processing Techniques for Electrical Capacitance Tomography Measurement System
}

\author{
Josiah Nombo $^{\mathbf{1}^{*}}$, Alfred Mwambela ${ }^{\mathbf{1}}$ and Michael Kisangiri ${ }^{2}$ \\ ${ }^{1}$ Electronics and Telecommunications Engineering Department, University of Dar es \\ Salaam, Tanzania \\ ${ }^{2}$ Electronics and Telecommunications Engineering Department, Nelson Mandela African \\ Institution of Science and Technology, Tanzania \\ *Corresponding author.E-mail addresses: jpnombo@gmail.com,jpnombo@udsm.ac.tz \\ Received 24 Nov 2020, Revised 1 Jul 2021, Accepted 20 Jul 2021, Published Aug 2021 \\ DOI: https://dx.doi.org/10.4314/tjs.v47i3.5
}

\begin{abstract}
To improve image quality generated from the electrical capacitance tomography measurement system, the use of entropic thresholding techniques is investigated in this article. Based on the analysis of the principle of Electrical Capacitance Tomography (ECT) image reconstruction and entropic thresholding, various algorithms have been proposed for easy extraction of quantitative information from tomograms generated from the ECT system. Experiments indicate that proposed algorithms can provide high-quality images at no or minimum computational cost. It is easier to implement and integrate with classical algorithms such as Linear Back Projection, Singular value decomposition, Tikhonov regularization, and Landweber. Entropic thresholding techniques present a feasible and effective way toward the industrial utilization of ECT measurement systems.
\end{abstract}

Keywords: Electrical Capacitance Tomography, Inverse Problem, Image Reconstruction, Entropic Thresholding.

\section{Introduction}

Electrical Capacitance Tomography (ECT) is a high-speed measuring technique that allows information about the operation of the process plant to be generated and presented as an image (Perera et al. 2017, Almutairi et al. 2020, Faia et al. 2020,). The idea is to measure the capacitances of a multi-electrode sensor surrounding an industrial vessel or pipe. These measurements are then used to reconstruct the cross-sectional image of the material being monitored. The generated image can then be used for industrial process diagnosis and control. ECT imaging, when compared with most other techniques, has drawn the considerable attention of researchers for it offers several merits, including a simplified structure, a wide range of applications, lower cost, non-destructive and non-invasive nature, and guaranteed safety (Chandrasekera et al. 2012). ECT image reconstruction relies on the measured capacitances and endures the following limitations: few measurements, softfield nature of the ECT sensor, non-linearity between measured capacitance and permittivity distribution, and severe ill-conditionedness caused by measurement errors (Zeeshan et al. 2019, Guo et al. 2020). Indeed, these weaknesses make solving the ECT image reconstruction problem a rather challenging task. 
Several image reconstruction methods have been proposed in the past years, with the popular ones being: Linear Back Projection (LBP) (Xie et al. 1992), Singular Value Decomposition (SVD) (Chen et al. 2010), Tikhonov regularization (Jinchuang et al. 2002, Soleimani and Lionheart 2005, Jing et al. 2009) Landweber and its variations (Yang et al. 1999, Lu et al. 2005, Jang et al. 2006), Conjugate gradient (Wang et al. 2005), and artificial neural networks and genetic algorithms (Warsito and Fan 2003, Changhua et al. 2005, Marashdeh et al. 2006).

The LBP is computationally efficient but suffers from larger reconstruction errors (Mwambela et al. 1997). The standard Tikhonov regularization whilst adequately addresses the ill-posed problem, its major weakness is to generate images with low resolutions (Yang and Peng 2003). Landweber iteration offers a good image reconstruction quality. However, it suffers from slow convergence rates, and thus unsuitable for online imaging environments (Lu et al. 2005). Artificial neural networks and genetic methods use the evolution of the initial estimate to select the best fitting data, which form an optimal solution. However, they prematurely converge a situation that makes them fail to reach a globally optimal solution (Zheng and Peng 2018, Guo et al. 2019, Zheng et al. 2019, Rymarczyk et al. 2019, 2021).

A different approach to improve the quality of the reconstructed images from the ECT system is to post-process the reconstructed images using image enhancement techniques (Xie et al. 1992, Mwambela et al. 1997, Mwambela and Johansen 2001, Nombo et al. 2014, Mwambela 2018). Image thresholding enhancement technique, which helps to stratify the multiphase-flow components (for example, oil and gas) has been investigated. Thresholding techniques are well-known and are the most efficient techniques in image enhancement due to their simplicity and fast processing speed. Entropic thresholding techniques were first proposed by Mwambela et al. (1997). In that work, entropic thresholding techniques based on the maximum entropy principle framework (Kapur et al. 1985) were implemented. Only two definitions of entropies; the Shannon and Pal entropy definitions were investigated. The performance of the resulting algorithms was evaluated using the LBP reconstruction method, which was the only available reconstruction algorithm in ECT at the time. It was established that algorithms using Shannon entropy were better than their counterpart using Pal entropy. The work was extended to include other more general definitions of entropy-Renyi and Tsallis, which addresses the limitations of the Shannon entropy as a source of information (Mwambela 2018). The authors underscored that incorporating entropic techniques in ECT image reconstruction significantly improves the image quality. In this study, the use of entropic thresholding techniques is extended to other conventional reconstruction methods evaluated for ECT systems to assess their performance. Specifically, entropic thresholding has been implemented along with LBP, SVD (Yan et al. 2004), Tikhonov (Tikhonov and Arsenin 1977, Jing et al. 2009), and Landweber (Yang et al. 1999, Lu et al. 2005, Jang et al. 2006) methods. The choice for these methods was based on their industrial applicability which includes high reconstruction speed and spatial efficiency.

Results show that thresholding techniques have improved spatial resolutions and the distribution error over a full component fractional range while saving the computational costs of timing errors. In particular, Kapurbased thresholding provides superior results for both annular and stratified flows.

\section{Materials and Methods \\ ECT measurement system}

ECT is an electronic measurement system, which comprises of three parts: sensor head, sensor electronics, and image reconstruction control and display units (Figure 1). 


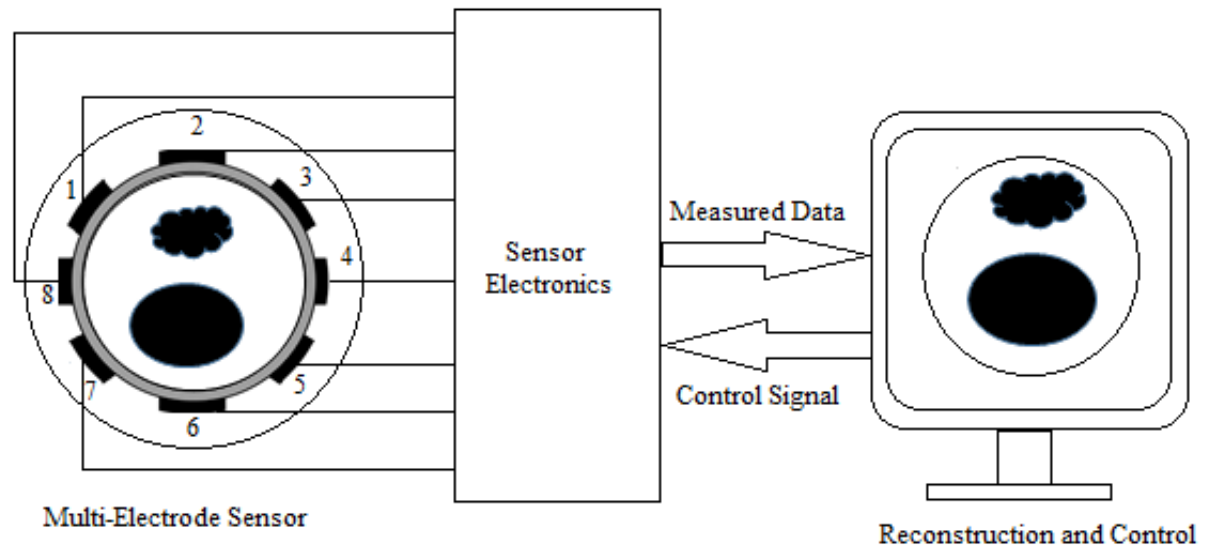

Figure 1: Basic components of an ECT system.

The ECT sensor consists of several electrodes, which are mounted around the process being monitored. A sensor with $n$ electrodes contains $\frac{n(n-1)}{2}$ possible combinations of electrode pairs and, therefore, $\frac{n(n-1)}{2}$ independent measurements of capacitance (Peng et al. 2011). The sensor electronics provide an electronic interface between the ECT sensor head and the image reconstruction unit, thus enabling the ECT sensor to interrogate the target process. The image reconstruction unit is a computer that controls the system and implements the associated methods.

\section{Image reconstruction problem in ECT}

The ECT image reconstruction process demands solving two computational problems, namely forward and inverse (Yang and Peng 2003). The forward problem, given by Equation 1

$$
C=S G
$$

is called a simplified model of the ECT system (Isaksen 1996), and calculates the capacitance values from a given permittivity distribution (image vector). From Equation $1, \mathrm{G}$ is an $N \times 1$ dimensional vector representing image vector, $\mathrm{N}$ is the number of pixels in the image, $\mathrm{C}$ represents an $M \times 1$ dimensional vector indicating the normalized capacitance values,
$M$ is the number of capacitance measurements, and $\mathrm{S}$ is an $M \times N$ field sensitivity matrix, which reflects the effect of permittivity distribution at each pixel on the inter-electrode capacitance.

The inverse problem attempts to calculate $G$ from the given capacitance data. In most cases, however, $\mathrm{S}$ is a non-square matrix that lacks a direct inverse, thus making the system prone to ill-posedness (Yang et al. 1999). Resulting in various reconstruction methods to address the problem.

\section{Image reconstruction algorithms in ECT}

Techniques to address inverse problems can be put into two groups: direct (single-step), where a single mathematical step is needed to directly generate the result from the measured capacitance and the sensitivity matrix; and iterative techniques, where a set of objective functions are optimized iteratively until steady conditions are encountered. Direct methods are computationally efficient but suffer from larger reconstruction errors compared to iterative ones. This section presents the conventional methods used to solve the ECT image reconstruction problem.

\section{Linear back projection}

In the LBP, the image vector is obtained through a linear mapping of the capacitance 
vector using the transpose of sensitivity matrix as given in Equation 2.

$$
G=S^{T} C \text {. }
$$

LBP performs better at a lower permittivity difference due to a smaller effect of the nonlinear interaction between pixels. At a higher permittivity difference, the effect of nonlinearity is higher, and consequently, LBP produces blurred images.

\section{Singular value decomposition (SVD)}

Due to the ill-posedness of the ECT inverse problem, it is challenging to directly invert the sensitivity matrix, and a pseudoinverse substitute method, which provides the least norm solution is preferred. Using the SVD method (Yan et al. 2004), an image can be obtained through a pseudoinverse process by using Equation 3;

$$
S=U \Sigma V^{T} .
$$

where columns of $U$ and $V$ are, respectively, formed by the eigenvectors of $S \times S^{T}$ and $S^{T} \times$ $S$, and $\Sigma$ is a diagonal matrix of the same size as $\mathrm{S}$ - which is formed by the square roots of the nonzero eigenvalues of both $S \times S^{T}$ and $S^{T} \times S$. Hence, the pseudoinverse of $\mathrm{S}$ is obtained by Equation 4

$$
\mathrm{S}^{+}=\mathrm{V} \Sigma^{-1} \mathrm{U}^{\mathrm{T}}
$$

and image reconstruction equation becomes (Equation 5)

$$
G=S^{+} C
$$

Tikhonov and iterative Tikhonov regularization

Tikhonov regularization uses the least-squares technique and introduces a regularization parameter into the formulation to solve the inverse problem (Jinchuang et al. 2002, Soleimani and Lionheart 2005). Therefore, the least-square form of Equation 1 is given in Equation 6

$$
S^{T} C=S^{T} S G,
$$

and $\mathrm{G}$ is calculated by Equation 7;

$$
G=\left(S^{T} S\right)^{-1} S^{T} C .
$$

In Equation 7, $S^{T} S$ is usually not invertible, which makes the system unstable and susceptible to multiple solutions; these issues may be addressed by incorporating a regularization parameter, $\mu$, as in Equation 8;

$$
G=\left(S^{T} S+\mu I\right)^{-1} S^{T} C,
$$

where I is the identity matrix. The quality of the reconstructed images depends strongly on the value of $\mu$. The Tikhonov method suffers from excessive smoothness, an effect that degrades the quality and spatial resolution of the reconstructed images (Jing et al. 2009). Addressing the weakness requires that the method is implemented iteratively, but at the expense of larger computational times, as in Equation 9

$$
\begin{gathered}
G_{k+1}=G_{k}+\left(S^{T} S+\mu I\right)^{-1} S^{T}(C \\
\left.-S G_{k}\right),
\end{gathered}
$$

and this is called the iterative Tikhonov regularization.

\section{Landweber and Projected Landweber}

Landweber iteration is an algorithm to solve linear inverse problems, and it has been extended to solve constrained non-linear problems. Proposed in the 1950s, the algorithm is currently regarded as a special case for many other general methods that solve inverse problems (Landweber 1951, Bauschke et al. 2011). Using the least of squares methods, Equation 1 can be modified to Equation 6. The Landweber algorithms (Yang et al. 1999) for solving Equation 6 is given by Equation 10;

$$
G_{k+1}=G_{k}-\alpha S^{T}\left(S G_{k}-C\right),
$$

where $\alpha$ is a relaxation factor, and $G_{k}$ is an initial image vector.

Although Landweber iteration is fast, it requires many iterations to achieve steady conditions (Jang et al. 2006). This makes the Landweber algorithm unsuitable for imaging in online industrial processes. To reduce computational times, a preconditioner matrix proposed by Strand (1974), can be applied, and the method is called preconditioned Landweber (Lu et al. 2005) as expressed in Equation 11 


$$
G_{k+1}=G_{k}-\alpha D S^{T}\left(S G_{k}-C\right),
$$

where $\mathrm{D}$ is a preconditioner matrix. For simplicity, D is normally chosen to be a diagonal matrix (Benzi 2002). To ensure convergence at every iteration step, Yang and Peng (2003) introduced a projection operator $\mathrm{P}$ to improve the overall convergence property of the Landweber iteration, and the algorithm is called Projected Landweber iteration as presented in Equation 12.

$$
G_{k+1}=P\left[G_{k}-\alpha S^{T}\left(S G_{k}-C\right)\right],
$$

$\mathrm{P}$ is defined by Equation 13 as

$$
P\left[G_{k}\right]=\left\{\begin{array}{l}
0 \text { if } G_{k}<0 \\
G_{k} \text { if } 0 \leq G_{k} \leq 1 \\
1 \text { if } G_{k}>1
\end{array}\right.
$$

The projection operator ensures that the reconstructed images are non-negative and upper-bounded. The Landweber algorithm is computationally efficient compared with other iteration algorithms as it uses first-order derivatives but suffers from a semiconvergence condition (Liu and Chang 2009) (image error decreases fast at the beginning of the iteration, but increases after reaching the local minimum point).

\section{Entropic image thresholding techniques}

One promising approach to enhance the quality of reconstructed images is to postprocess tomograms using thresholding techniques, which involve binarization for easy interpretation, analysis, and processing (Mwambela 2018). With this technique, we can cheaply segment the contents of tomograms into two components of interest (oil and gas). The proposed ECT system that incorporates the thresholding process is depicted in Figure 2.

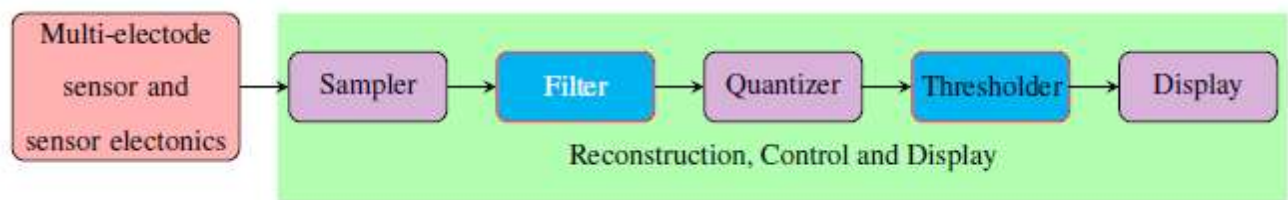

Figure 2: ECT system incorporating data fitting and thresholding processes.

The ECT system in Figure 2 is an extended version of the one proposed by Mwambela et al. (1997). It consists of two main parts: hardware - which consists of a multi-electrode sensor and sensor electronics - and software that holds an imaging program, which implements sampler, filter, quantizer, and thresholder. The sampler uses the sensitivity matrix to convert measured capacitance data to grey levels (which constitute a discretecontinuous image); filter removes extraneous noise in the measured capacitance; quantizer converts a discrete-continuous image into a discrete-discrete image by spanning the image data with the selected quantizer resolution as the multiplying factor, then rounding to the nearest integer; and thresholder converts the quantized image into a new simple discretediscrete image, whose image values are binarized (0 and 1). For the case of the oil-gas tomogram, the basic assumption is that oil and gas pixels in the tomogram can be distinguished by their grey levels. By carefully selecting a thresholding grey level between dominant values of oil and gas intensities, the original grey level tomogram can be transformed into a binary image so that the tomogram pixels associated with the oil and gas will assume values of one and zero, respectively.

Automatically selecting the threshold grey level is critical in industrial automation and control. The challenge, however, is to find a thresholding function that can self-segment the required tomograms of interest. In this work, we have adopted the classical thresholding techniques based on the principle of maximum entropy (Chang and Chong-xu 2012, Sarkar and Das 2013, Sarkar et al. 2015) as they are simple, fast, and more efficient. In this section, a theoretical background of some superior thresholding algorithms is given. 
Consider an image with $N$ pixels, $\mathrm{n}$ grey levels, and frequency distribution, $f_{i}$, of the $i^{\text {th }}$ grey level, such that $\sum_{i=1}^{n} f_{i}=N$.

\section{Renyi entropy}

The entropy of a tomogram based on Renyi entropy is defined by Equation 14;

$$
\begin{aligned}
H_{n} & \frac{1}{(1-q)} \ln \left[\frac{\sum_{i=1}^{n}\left(p_{i}\right)^{q}}{\sum_{i=1}^{n}\left(p_{i}\right)}\right],
\end{aligned}
$$

where $p_{i}=\frac{f_{i}}{N}$ is the probability of occurrence of the $i^{\text {th }}$ grey level, $q_{i} \neq 1$ and $q_{i}>0$.

\section{Shannon entropy}

When $q_{i} \rightarrow 1$ the Equation 14 reduce to Shannon definition of entropy

$$
=\sum_{i=1}^{H_{n}} \ln \left(p_{i}\right)
$$

This Equation 15 is not defined at $p_{i}=0$, as a result, Pal proposed a new definition to address this problem.

\section{Pal entropy}

$$
H_{n}=\sum_{i=1}^{n} p_{i} \exp \left(1-p_{i}\right),
$$

$$
\begin{aligned}
H_{n}=\frac{1}{(q-1)}\left(\begin{array}{l}
1 \\
\\
\end{array} \quad-\sum_{i=1}^{n}\left(p_{i}\right)^{q}\right),
\end{aligned}
$$

The use of maximum entropy principles yields the same results for both Renyi and Tsallis entropic thresholding. It can be shown that the two entropy definitions are monotonic functions of each other.

In this work, Shannon and Renyi entropy definitions have been used to determine the maximum entropy of tomograms as presented in Equation 18 to Equation 21.

$$
H_{n}(T)=H_{o}(T)+H_{b}(T)
$$

$$
\begin{gathered}
H_{o}(T)=\frac{1}{(1-q)} \ln \left[\sum_{i=1}^{T}\left(\frac{p_{i}}{P_{T}}\right)^{q}\right] \\
H_{b}(T)=\frac{1}{(1-q)} \ln \left[\sum_{i=T+1}^{n}\left(\frac{p_{i}}{\left(1-P_{T}\right)}\right)^{q}\right] \\
T_{o p t}=\arg \max \left[H_{o}(T)+H_{b}(T)\right]
\end{gathered}
$$

Various algorithms have been designed based on the building blocks presented in Figure 2. For convenience, the algorithms at each stage of reconstruction are summarized in Table 1.

Tsallis entropy

Table 1: List of implemented algorithms at sampler and thresholder stages

\begin{tabular}{llll}
\hline Algorithm name & Sampler & $\begin{array}{l}\text { Shannon } \\
\text { Thresholder Kapur }\end{array}$ & $\begin{array}{l}\text { Renyi } \\
\text { Thresholder Pal }\end{array}$ \\
\hline Linear back projection & LBP & LBPS & LBPR \\
Singular value decomposition & SVD & SVDS & SVDR \\
Tikhonov regularization & TIK & TIKS & TIKR \\
Iterative Tikhonov regularization & ITIK & ITIKS & ITIKR \\
Landweber & LAND & LANDS & LANDR \\
Projected Landweber & PLAND & PLANDS & PLANDR \\
\hline
\end{tabular}

\section{Evaluation criteria}

To evaluate the performance of the algorithms, experiments were conducted using a circular sensor ECT system with eight electrodes (excitation waveform:
$10 \mathrm{Vpp}, 300-500 \mathrm{kHz}$ ), uniformly spaced and arranged around the vessel. The imaging area was divided into 900 pixels. MATLAB software was used for image reconstruction and presentation. Annular bubble and stratified 
flows were used to evaluate the algorithms. These flows represent the gas-oil distribution frequently encountered in oil industries.

Qualitative and quantitative metrics were used to evaluate the efficacy of various methods. In the former evaluation metric, visual results of the reconstructed images generated by different algorithms were subjectively compared. To quantify the quality of results, image distribution error (DE) was used to compare the spatial resolution between the reference and the reconstructed images as defined by Equation 26 (Isaksen and Nordtved 1994)

$$
D E=\frac{1}{N} \sum_{i=1}^{N}\left|G_{i}^{r e c}-G_{i}^{r e f}\right| .
$$

where $G_{i}^{r e c}$ and $G_{i}^{r e f}$ are, respectively, the reference and reconstructed image vectors for an element $i$, and $N$ is the total number of pixels. Lower DE signals better results; for reservoir management in oil industries, for example, the desired DE should be less or equal to $10 \%$.

\section{Results and Discussions}

Figures 3 to 8 , present qualitative evaluations of algorithms implemented at the sampler and the thresholder stages. The reconstructions are based on actual capacitance measurements of dielectric objects located at different positions of the sensing domain. Each row in every figure contains a reference image, and reconstructions at the sampler, and thresholder stages. Figure 3 shows the reconstruction results based on the LBP, LBPS, and LBPR. Results show an improvement in the quality of images generated using LBPS and LBPR compared with those of LBP for all three test objects. The thresholding method based on Reny entropy is superior to Shannon entropy for annular, and bubble flows.

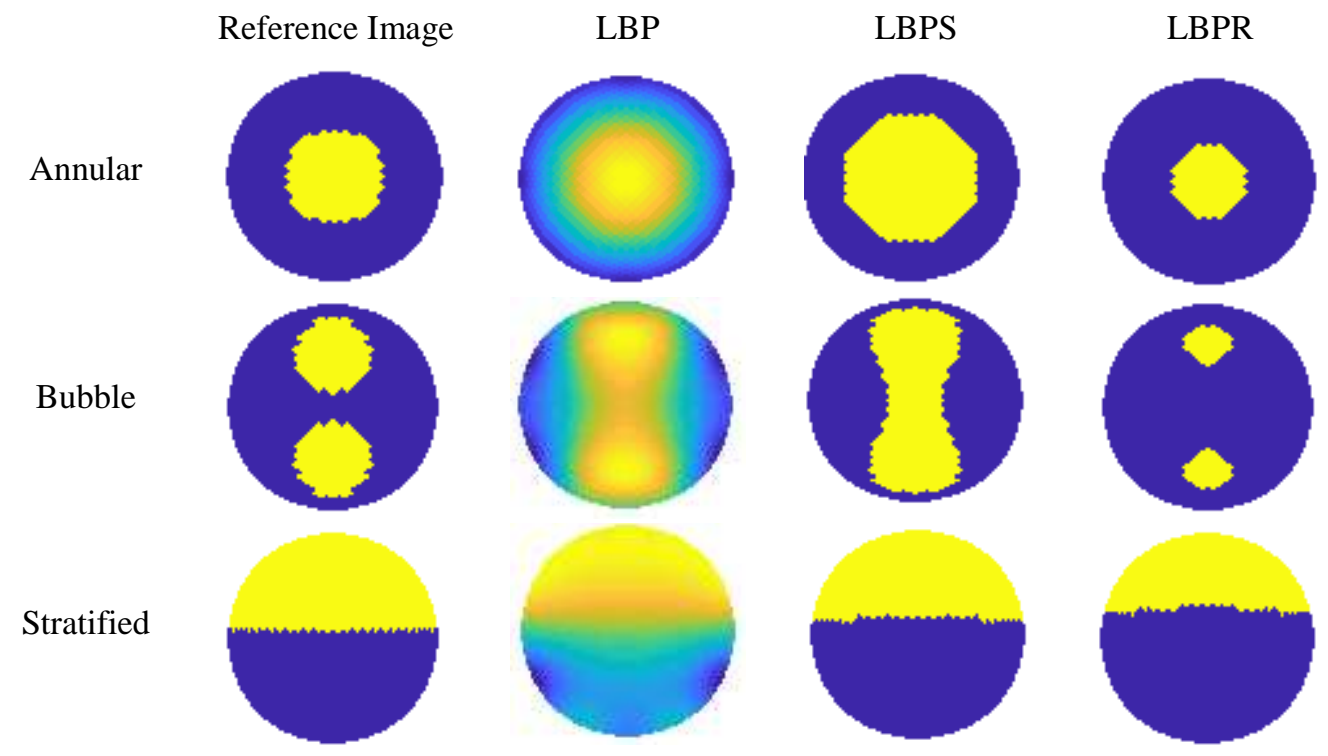

Figure 3: Images reconstructed using LBP, LBPS, and LBPR. 


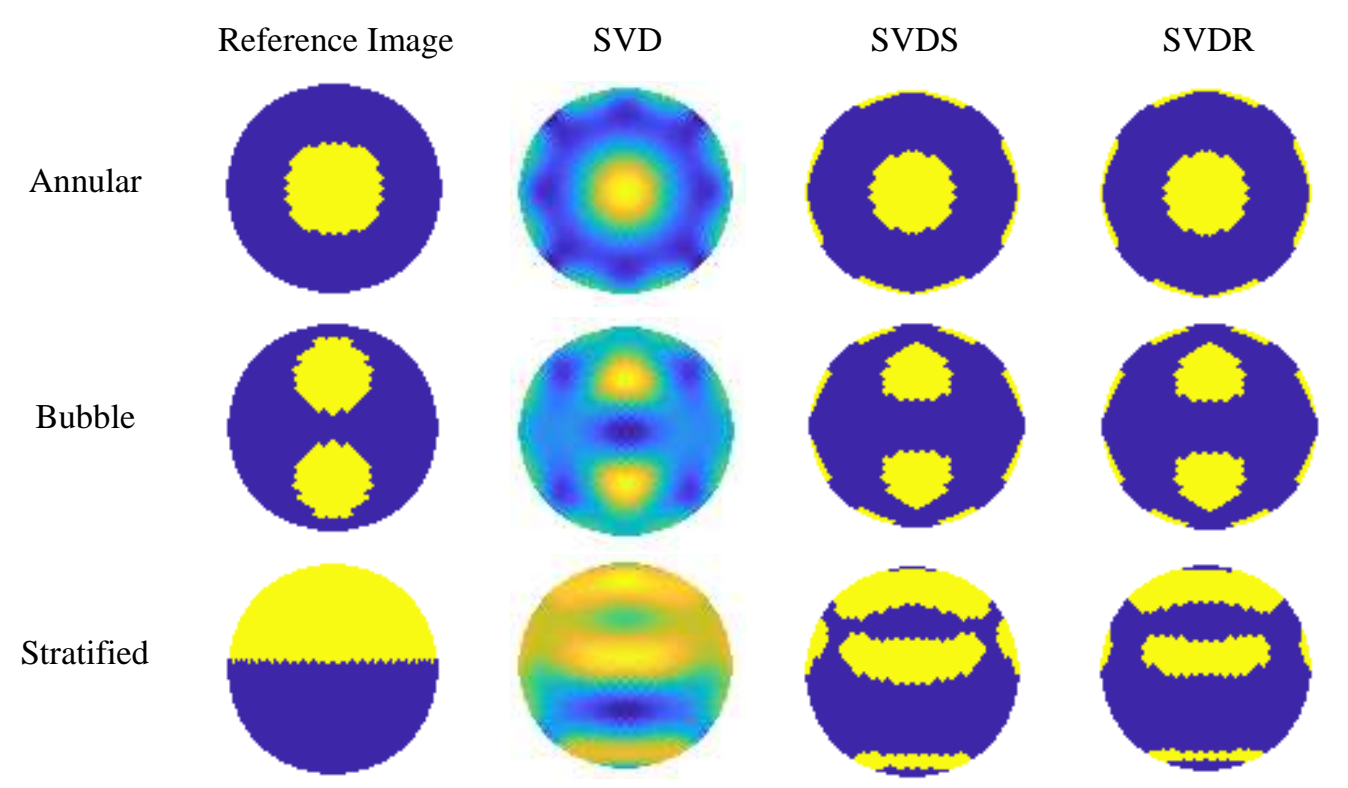

Figure 4: Images reconstructed using SVD, SVDS, and SVDR.

In Figure 4 reconstruction results using SVD and SVDS, and SVDR are presented. The reconstructed images are distorted near the sensor walls and have a better resolution at the center for all three flows. This is due to artifacts in the pseudoinverse of the sensitivity matrix used in grey levels calculation when using SVD at the sampler stage.

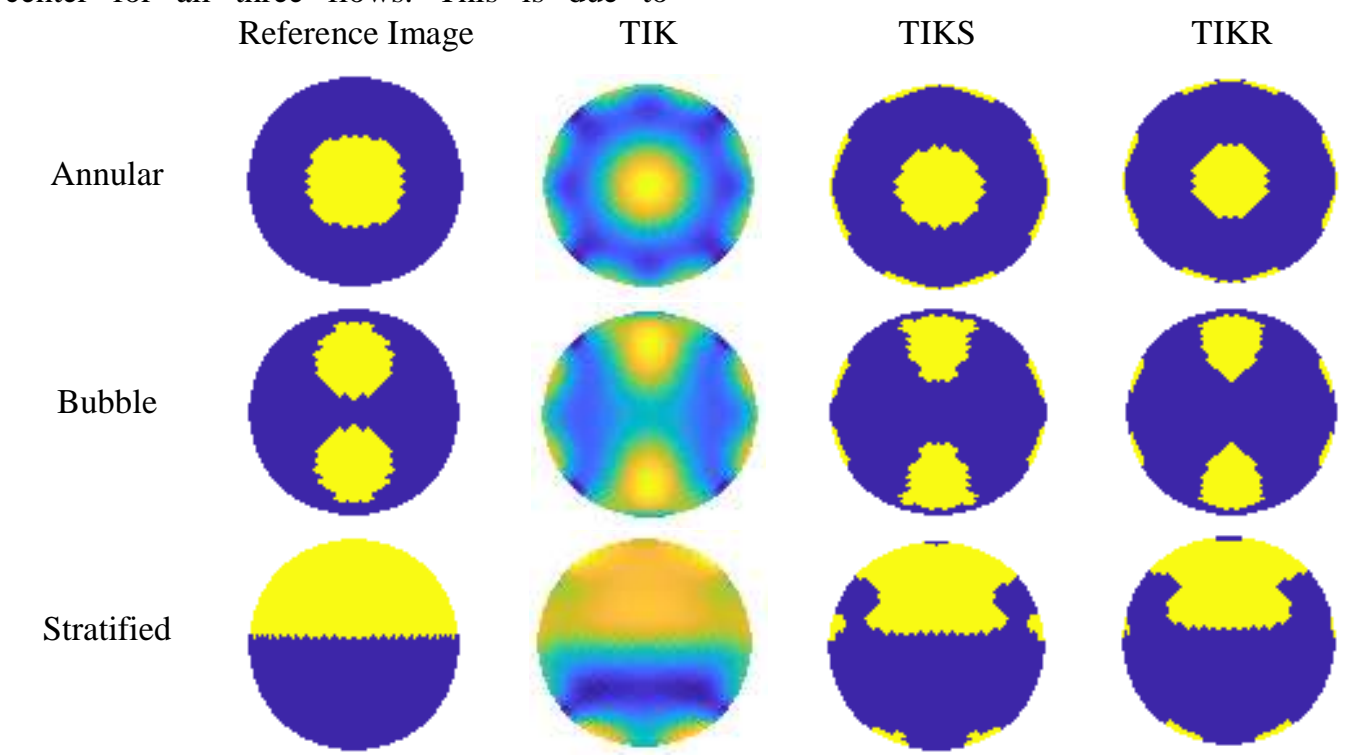

Figure 5: Images reconstructed using TIK, TIKS, and TIKR. 
Figure 5 shows the results of images generated using TIK, TIKS, and TIKR methods. Both TIKS and TIKR generate deformed imaged at the sensor walls and near

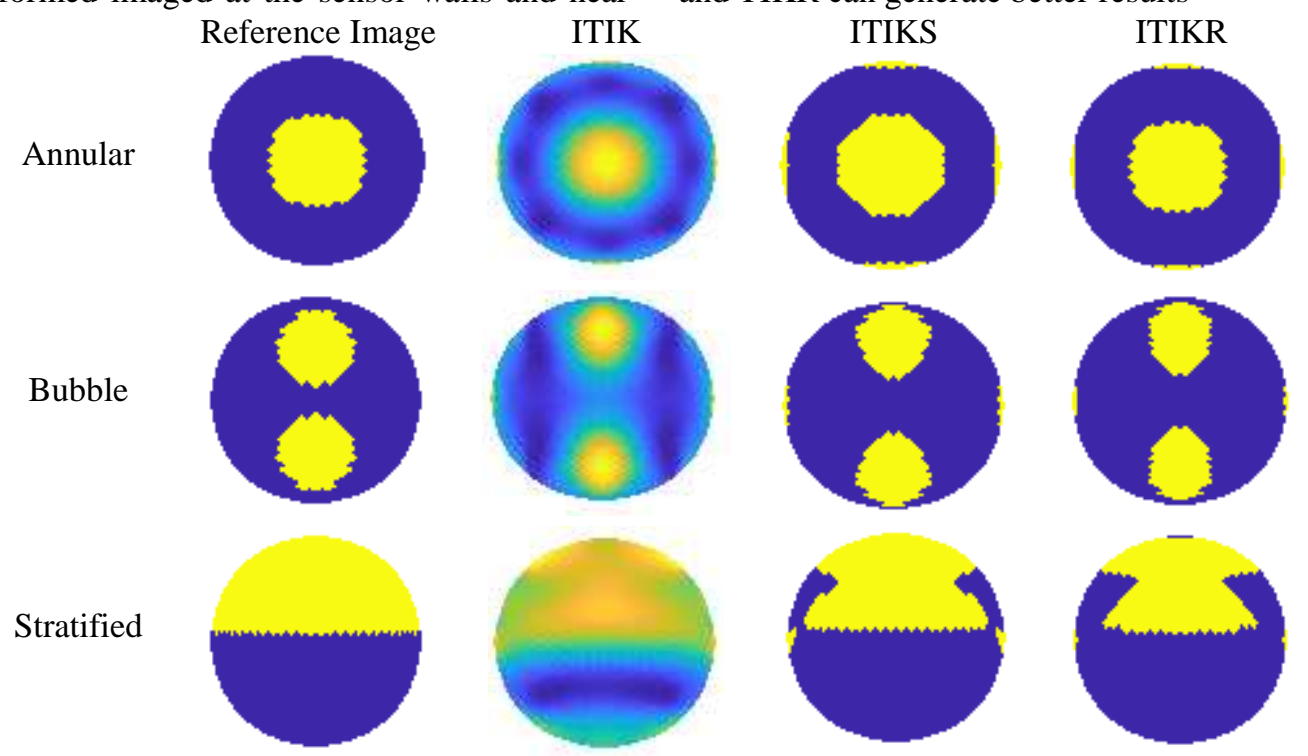

Figure 6: Images reconstructed using ITKR, ITKRK, and ITKRP.

Figure 6 presents images reconstructed by ITIK, ITIKS, and ITIKR. ITIKS generates visually appealing images for all flows. ITIKR produces better images for stratified compared
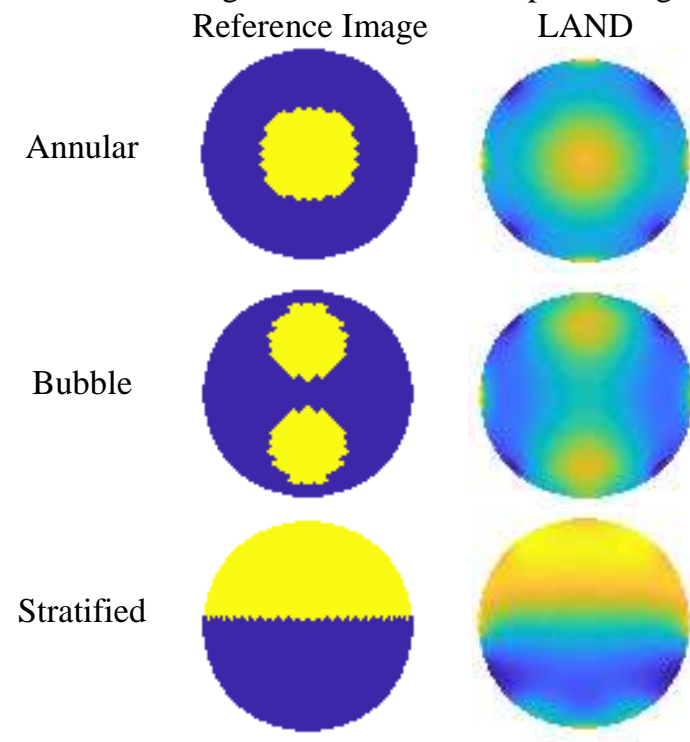

to annular and bubble flows. The choice of initial grey vector and regularization parameter is important for ITIK based thresholders to generate better images.

$$
\text { LANDS }
$$
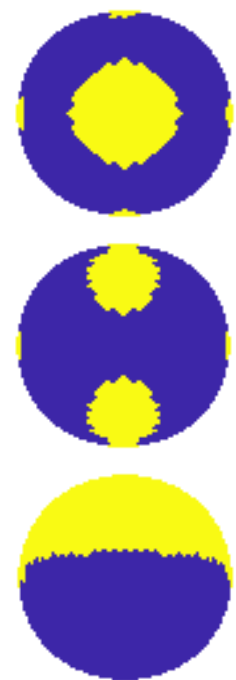

LANDR
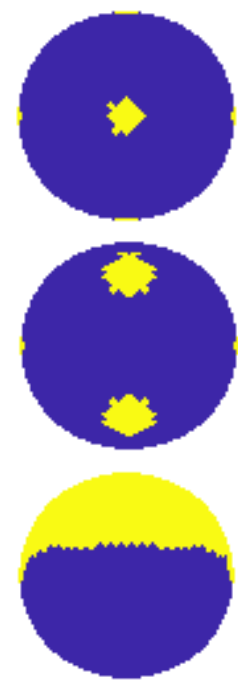

Figure 7: Images reconstructed using LAND, LANDS, and LANDR. 


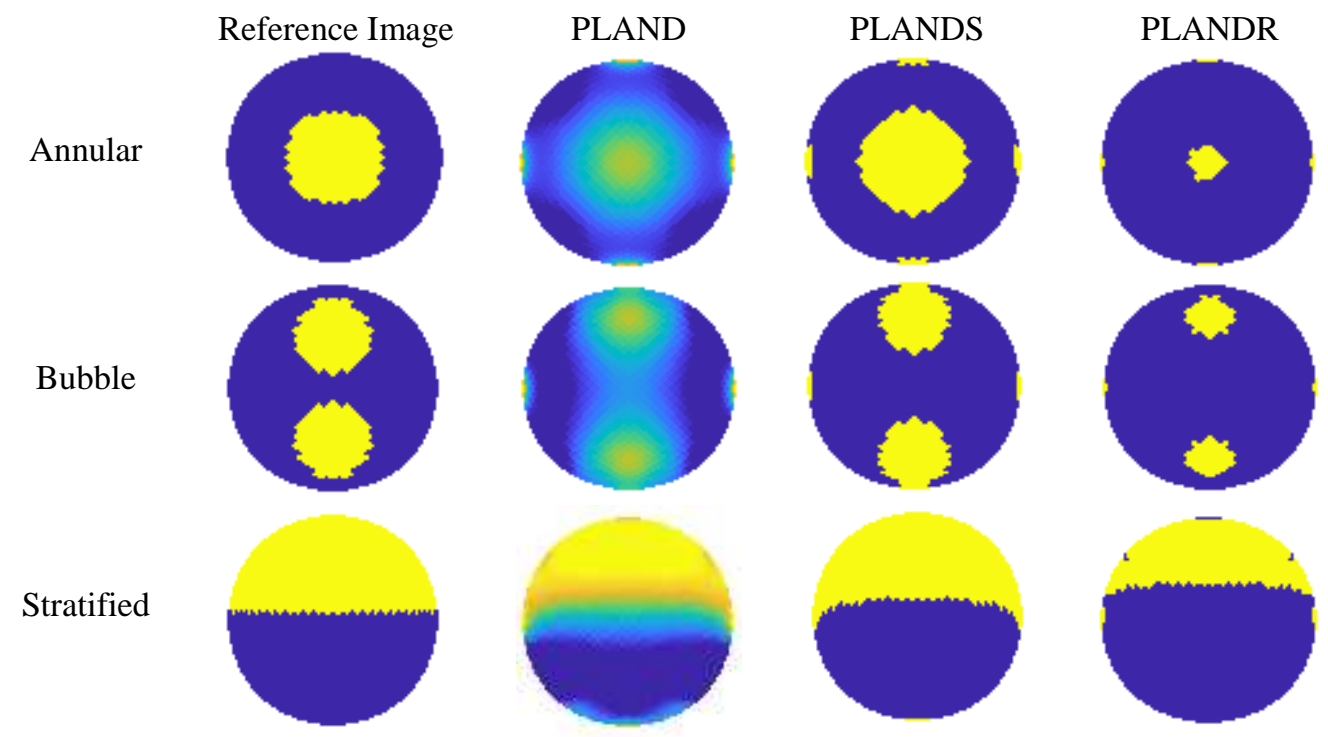

Figure 8: Images reconstructed using PLAND, PLANDS, and PLANDR.

Visual results generated from LAND, LANDS, LANDR are shown in Figure 7. The results show that LANDS generate visually appealing images which are sharper and more detailed compared to LANDR. For annular, and bubble flows. Figure 8 shows the reconstruction results from PLAND, PLANDS, and PLANDR. Results show an improvement in the quality of images generated using PLANDS compared with those of PLANDP and PLAND for all three test objects.

Qualitative visual inspection shows that thresholding algorithms have better resolutions than conventional ones. In particular, Shannonbased methods produced better images for all flows. However, the quality of reconstructed images depends on the choice of the algorithm at the sampler stage. In particular, poor results are observed for the sampler algorithms. This is attributed to the nonlinear relationship between capacitance measurements and permittivity distribution and the limited number of independent measurements. On the contrary, the thresholder algorithms generate better images because of the careful automatic selection of the thresholding level. This can simplify decision-making when multiphase classifying components in multiphase flows.

Figure 9 to Figure 16 present quantitative results based on $\mathrm{DE}$ for the algorithms implemented at the sampler and thresholder stages. Although the quantizer algorithms have been implemented, their qualitative results have not been presented, because they perform similar to corresponding sampler algorithms. 


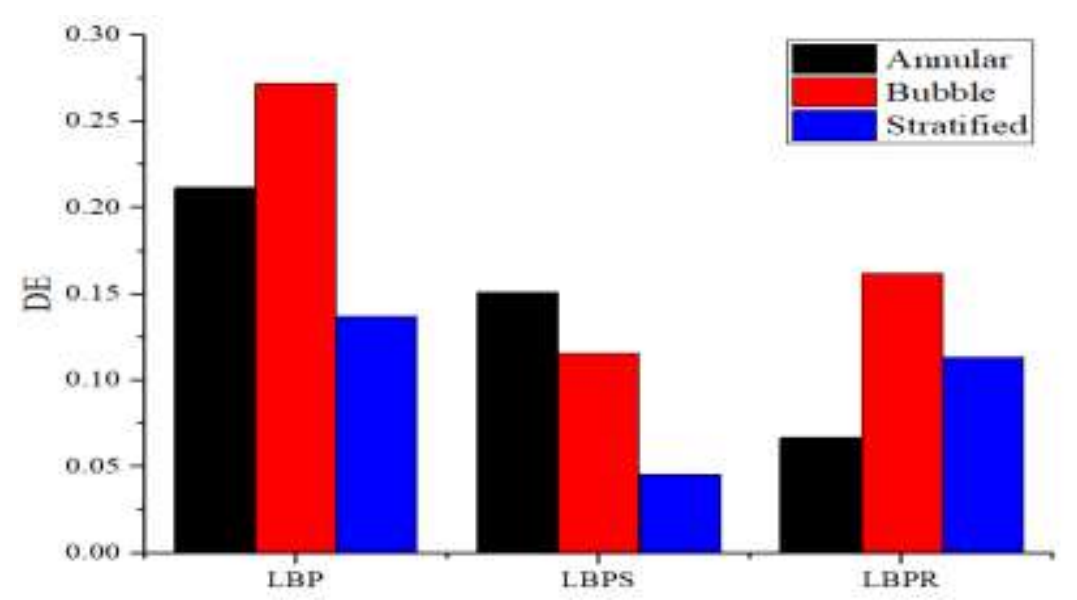

Figure 9: DE for LBP, LBPS, and LBPR for annular, bubble, and stratified flows.

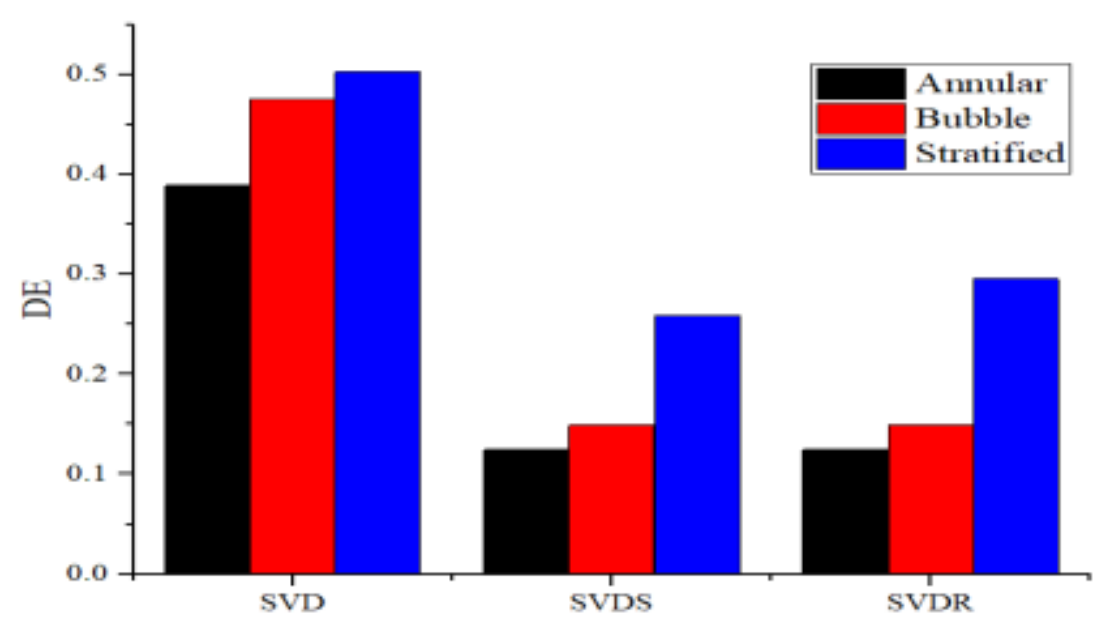

Figure 10: DE for SVD, SVDS, and SVDR for annular, bubble, and stratified flows.

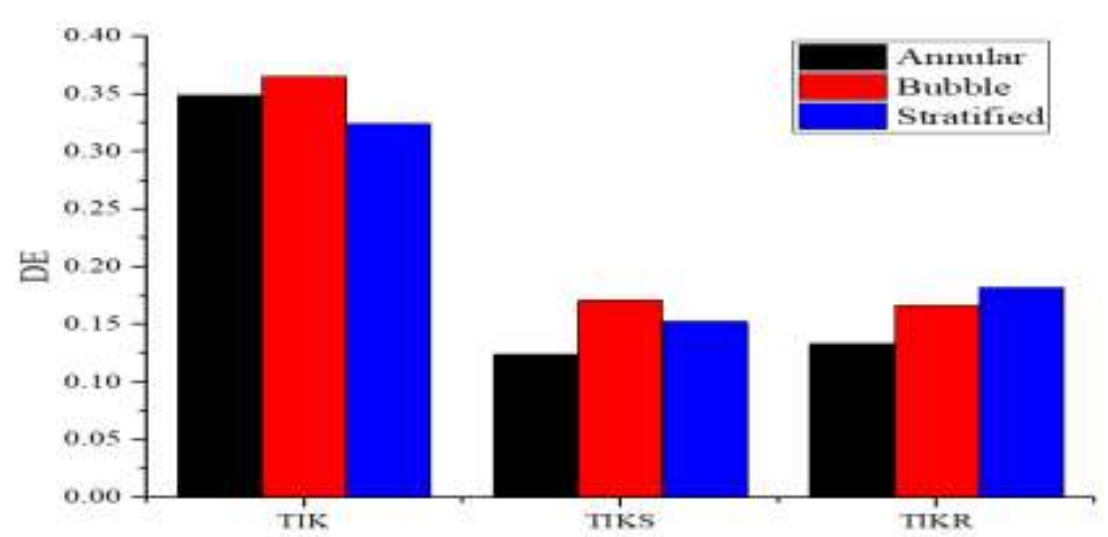

Figure 11: DE for TIK, TIKS, and TIKR for annular, bubble, and stratified flows. 


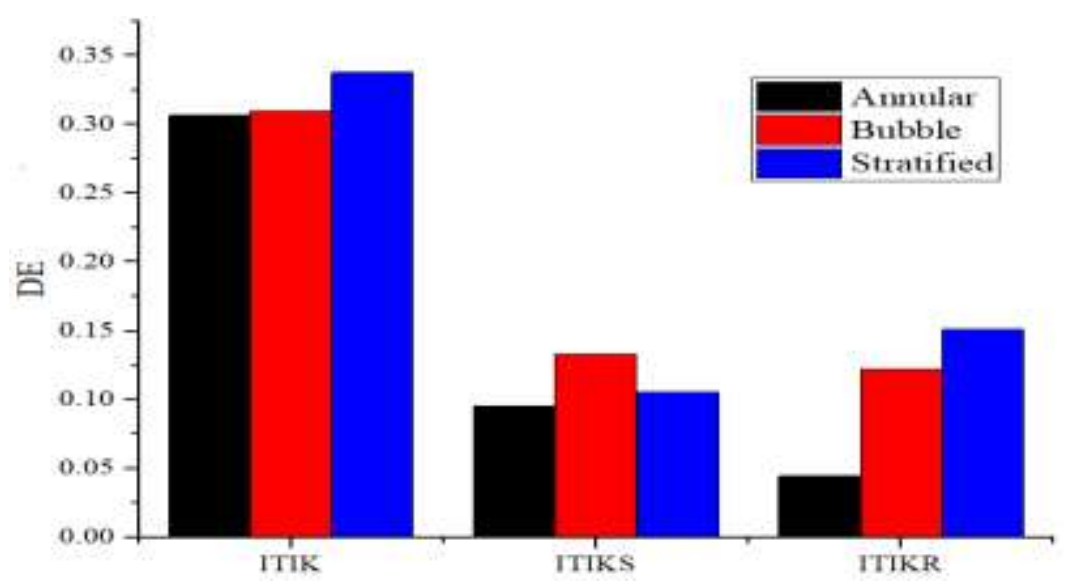

Figure 12: DE for ITIK, ITKS, and ITIKR for annular, bubble, and stratified flows.

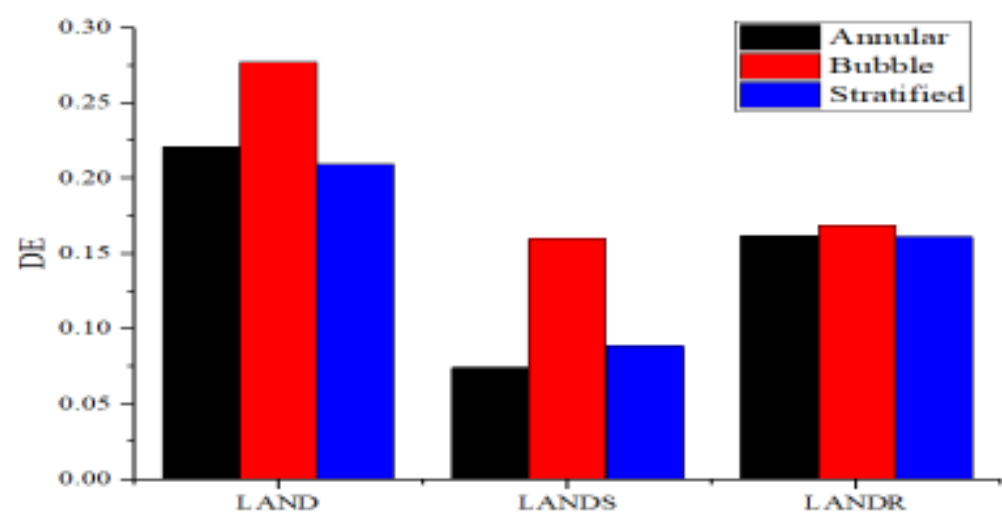

Figure 13: DE for LAND, LANDS, and LANDR for annular, bubble, and stratified flows.

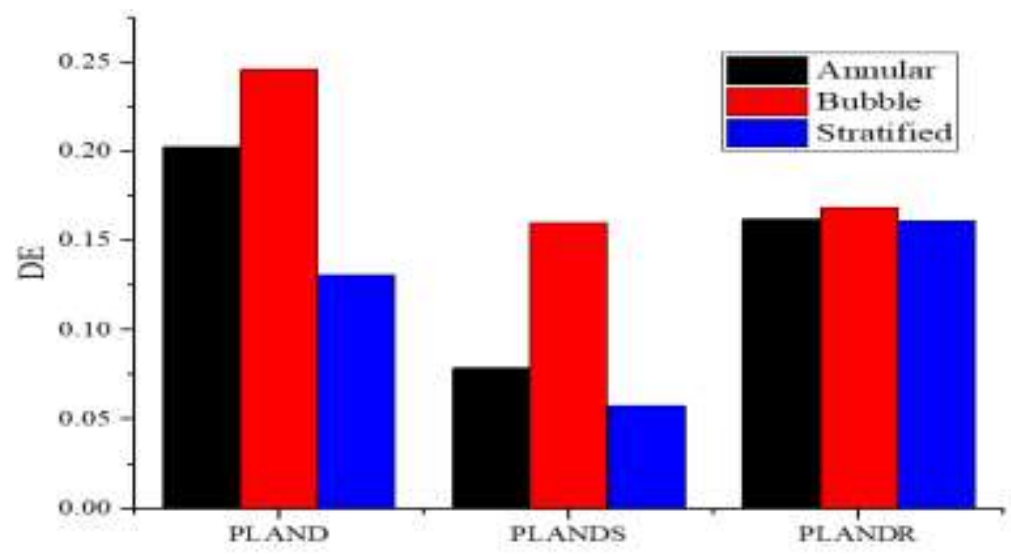

Figure 14: DE for PLAND, PLANDS, and PLANDR for annular, bubble, and stratified flows. 
Quantitatively, it is noted that algorithms implementing the thresholding stage are more accurate compared with sampler algorithms (See Figure 9 to Figure 14). The results emphasize a significant difference between the spatial similarity performances of the thresholder algorithms compared with sampler algorithms. In general, the above analysis suggests that Shannon-based thresholder algorithms significantly improve the quality of reconstructed images.

\section{Conclusions}

In this paper, image thresholding techniques based on the principle of maximum entropy have been proposed to assist the extraction of quantitative information from noisy capacitance data obtained from the ECT measurement system. The methods have been evaluated for their suitability to estimate the component fraction in the industrial oil-gas multiphase flow. Results suggest that thresholding improves the interpretability of the images. However, its performance is influenced by the reconstruction used. In particular, Shanon-based thresholders produce better qualitative and quantitative results than Renyi for most of the evaluated thresholding algorithms. PLAND and LAND are the most accurate iterative algorithms than LBP. However, if the speed of reconstruction is important LBP offer a better compromise to accuracy and may be considered as a step forward to apply ECT systems for quantitative analysis in industrial applications.

\section{Acknowledgments}

The author appreciates assistance from anonymous referees for their helpful comments for the improvement of the paper.

\section{References}

Almutairi Z, Al-Alweet FM, Alghamdi YA, Almisned OA, and Alothman OY 2020 Investigating the characteristics of twophase flow using electrical capacitance tomography (ECT) for three pipe orientations. Processes 8(1): 78.
Bauschke H, Burachik R, and Combettes P, Elser V, Luke DR and Wolkowicz H (Eds) 2011 Fixed-Point Algorithms for Inverse Problems in Science and Engineering. Springer.

Benzi M 2002 Preconditioning techniques for large linear systems: a survey. J. Computat. Phys. 182(2): 418-477.

Chandrasekera T, Wang A, Holland D, Marashdeh Q, Pore M, Wang F, Sederman A, Fan L, Gladden L and Dennis J 2012 A comparison of magnetic resonance imaging and electrical capacitance tomography: An air jet through a bed of particles. Powder Technol. 227: 86-95.

Chang L and Chong-xu Y 2012 Image segmentation based on maximum entropy and kernel self-organizing map. In Engineering and Technology (S-CET), 2012 Spring Congress on Engineering and Technology, pp. 1-4. IEEE.

Changhua M, Lihui P, Danya Y and Deyun X 2005 Image reconstruction using a genetic algorithm for electrical capacitance tomography. Tsinghua Sci. Technol. 10(5): 587-592.

Chen Y, Gao B, Zhang GL, Chen D and Yu X 2010 Image reconstruction based on weighted SVD truncation conjugate gradient algorithm for electrical capacitance tomography. Optics Precis. Eng. 18(3): 701-707.

Faia P, Silva R, Rasteiro MG, and Garcia F 2020 Electrical tomography: a review of configurations, and application to fibre flow suspensions characterisation. Appl. Sci. 10(7): 2355

Guo Q, Li X, Hou B, Mariethoz G, Ye M, Yang W, and Liu Z 2020 A novel image reconstruction strategy for ECT: combining two algorithms with a graph cut method. IEEE Trans. Instrum. Meas. 69(3): 804814.

Guo Q, Ye M, Yang W, and Liu Z 2019 A machine learning approach for electrical capacitance tomography measurement of gas-solid fluidized beds. AIChE J. 65(6): e16601. 
Isaksen Ø 1996 A review of reconstruction techniques for capacitance tomography. Meas. Sci. Technol. 7(3): 325-337.

Isaksen $\varnothing$ and Nordtvedt JE 1994 An implicit model based reconstruction algorithm for use with a capacitance tomography system. In Proc. European Concerted Action on Process Tomography, Oporto 215-226.

Jang JD, Lee SH, Kim KY, and Choi BY 2006 Modified iterative Landweber method in electrical capacitance tomography. Meas. Sci. Technol. 17(7): 1909-1917.

Jinchuang Z, Wenli F, Taoshen L, and Shi W 2002 An image reconstruction algorithm based on a revised regularization method for electrical capacitance tomography. Meas. Sci. Technol. 13(4): 638-640.

Jing L, Liu S, Zhihong L, and Meng S 2009 An image reconstruction algorithm based on the extended Tikhonov regularization method for electrical capacitance tomography. Measurement 42(3): 368-376.

Kapur J, Sahoo P, and Wong A 1985 A new method for gray-level picture thresholding using the entropy of the histogram. Computer Vision, Graphics, and Image Processing. 29: 273-285.

Landweber L 1951 An iteration formula for Fredholm integral equations of the first kind. Am. J. Math. 73(3): 615-624.

Liu C and Chang C 2009 Novel methods for solving severely ill-posed linear equations system. J. Marine Sci. Tech. 17: 216-227.

Lu G, Peng L, Zhang B, and Liao Y 2005 Preconditioned Landweber iteration algorithm for electrical capacitance tomography. Flow Meas. Instrum. 16(2-3): 163-167.

Marashdeh Q, Warsito W, Fan L-S, and Teixeira FL 2006 A nonlinear image reconstruction technique for ECT using a combined neural network approach. Meas. Sci. Technol. 17(8): 2097-2103.

Mwambela AJ, Isaksen $\varnothing$, and Johansen G 1997 The use of entropic thresholding methods in reconstruction of capacitance tomography data. Chem. Eng. Sci. 52(13): 2149-2159.
Mwambela AJ and Johansen GA 2001 Multiphase flow component volume fraction measurement: experimental evaluation of entropic thresholding methods using an electrical capacitance tomography system. Meas. Sci. Technol. 12(8): 10921101.

Mwambela AJ 2018 Comparative performance evaluation of entropic thresholding algorithms based on Shannon, Renyi and Tsallis entropy definitions for electrical capacitance tomography measurement systems. Int. J. Intell. Syst. Applic. 10: 4149.

Nombo J, Mwambela AJ and Kisangiri M 2016 A review of image reconstruction methods in electrical capacitance tomography. $J$. Math. Comput. Sci. 6(1): 39-57.

Nombo J, Mwambela A and M K 2014 A grey level fitting mechanism based on Gompertz function for two phase flow imaging using electrical capacitance tomography measurement. Int. J. Comput. Applic. 101(8): 7-12.

Peng L, Ye J, Lu G, and Yang W 2011 Evaluation of effect of number of electrodes in ECT sensors on image quality. IEEE Sensors J. 12(5): 1554-1565.

Perera K, Pradeep C, Mylvaganam S, and Time RW 2017 Imaging of oil-water flow patterns by electrical capacitance tomography. Flow Meas. Instrum. 56: 2334.

Rymarczyk T, Kłosowski G, Hoła A, Sikora J, Wołowiec T, Tchórzewski P, and Skowron S 2021 Comparison of machine learning methods in electrical tomography for detecting moisture in building walls. Energies 14(10): 2777.

Rymarczyk T, Klosowski G, Kozlowski E, Rymarczyk P, and Niderla K 2019 Process analysis with electrical impedance and capacitance tomography data. In 2019 Applications of Electromagnetics in Modern Engineering and Medicine, PTZE 2019, 161-165. Institute of Electrical and Electronics Engineers Inc.

Sarkar S and Das S 2013 Multilevel image 
thresholding based on 2D histogram and maximum Tsallis entropy-a differential evolution approach. Image Process. IEEE Trans. Image Process. 22(12): 4788-4797.

Sarkar S, Das S, and Chaudhuri S 2015 A multilevel color image thresholding scheme based on minimum cross entropy and differential evolution. Pattern Recognit. Lett. 54: 27-35.

Soleimani M and Lionheart WWRB 2005 Nonlinear image reconstruction for electrical capacitance tomography using experimental data. Meas. Sci. Technol. 16(10): 1987-1996.

Strand O 1974 Theory and methods related to the singular-function expansion and Landweber's iteration for integral equations of the first kind. SIAM J. Numerical Anal. 11(4): 798-825.

Tikhonov A and Arsenin V 1977 Solutions of ill-posed problems. WH Winston, Washington, DC 330.

Wang H, Zhu X, and Zhang L 2005 Conjugate gradient algorithm for electrical capacitance tomography. Tianjin Daxue Xuebao (J. Tianjin Univ. Sci. Technol.) 38(1): 1-4.

Warsito W and Fan L 2003 Neural network multi-criteria optimization image reconstruction technique (NN-MOIRT) for linear and non-linear process tomography. Chem. Eng. Process.: Process Intensification 42(8-9): 663-674.

Xie CG, Huang SM, Beck MS, Hoyle BS, Thorn R, Lenn C, and Snowden D 1992 Electrical capacitance tomography for flow imaging: system model for development of image reconstruction algorithms and design of primary sensors. IEE Proceedings $G$
(Circuits, Devices and Systems). 139(1): 89-98.

Yan H, Liu C and Gao J 2004 Electrical capacitance tomography image reconstruction based on singular value decomposition. In Fifth World Congress on Intelligent Control and Automation (IEEE Cat. No.04EX788) Vol. 4, 3783-3786. IEEE.

Yang W and Peng L 2003 Image reconstruction algorithms for electrical capacitance tomography. Meas. Sci. Technol. 14(1): R1-R13.

Yang W, Spink D, York TA and McCann H 1999 An image-reconstruction algorithm based on Landweber's iteration method for electrical-capacitance tomography. Meas. Sci. Technol. 10(11): 1065-1069.

Zeeshan Z, Zuccarelli CE, Acero DO, Marashdeh QM, and Teixeira FL 2019 Enhancing resolution of electrical capacitive sensors for multiphase flows by fine-stepped electronic scanning of synthetic electrodes. IEEE Trans. Instrum. Meas. 68(2): 462-473.

Zheng J, Ma H, and Peng L 2019 A CNNbased image reconstruction for electrical capacitance tomography. In IST 2019 IEEE International Conference on Imaging Systems and Techniques, Proceedings. Institute of Electrical and Electronics Engineers Inc.

Zheng J and Peng L 2018 An autoencoderbased image reconstruction for electrical capacitance tomography. IEEE Sensors J. 18(13): 5464-5474. 